\title{
DNA Adduct Assessment During Antihormonal Treatment of Perianal Gland Tumors With Tamoxifen in Male Dogs
}

\author{
ADAM BRODZKI ${ }^{1}$, MARCIN R. TATARA $^{2}$, PIOTR BRODZKI $^{3}$ and IRENEUSZ BALICKI ${ }^{1}$ \\ ${ }^{1}$ Department and Clinic of Animal Surgery, Faculty of Veterinary Medicine, \\ University of Life Sciences in Lublin, Lublin, Poland; \\ ${ }^{2}$ Department of Animal Physiology, Faculty of Veterinary Medicine, \\ University of Life Sciences in Lublin, Lublin, Poland; \\ ${ }^{3}$ Department and Clinic of Animal Reproduction, Faculty of Veterinary Medicine, \\ University of Life Sciences in Lublin, Lublin, Poland
}

\begin{abstract}
Background/Aim: Determination of DNA adduct count was performed in mononuclear cells during antihormonal treatment of perianal gland tumors. Materials and Methods: Eight- to fifteen-year-old dogs with carcinoma (CAR Group; N=5), epithelioma (EPI Group; N=16) or adenoma (ADE Group; N=24) were used. The control group suffered from perineal hernia or rectal diverticulum (CTR Group; N=25). Blood was collected at baseline, and at one and six months after the beginning of the anti-hormonal treatment with tamoxifen (1 $\mathrm{mg} / \mathrm{kg}$ of body weight). DNA adduct count was determined using autoradiography. Results. At baseline, DNA adduct count reached the highest value in the CTR Group, and the lowest in the EPI Group $(p<0.05)$. Sixmonth-long therapy with tamoxifen resulted in a significant increase in the DNA adduct count by $78.7 \%, 221.5 \%$ and $198.3 \%$ in the ADE, EPI and CAR groups, respectively $(p<0.05)$. Conclusion: Increased DNA adduct formation after long-term administration of tamoxifen shows its genotoxicity.

Perianal glands in dogs are considered as modified sebaceous glands located in the skin of the perianal region, perineum, prepuce, internal femoral region, lower surface of the tail and occasionally in other regions of the body. The development of perianal gland tumors is associated with stimulatory action of androgens and estrogens (1). Perianal gland hyperplasia
\end{abstract}

This article is freely accessible online.

Correspondence to: Adam Brodzki, Department and Clinic of Animal Surgery, Faculty of Veterinary Medicine, University of Life Sciences in Lublin, ul. Głęboka 30, 20-612 Lublin, Poland. Tel: +48 814456207, Fax: +48 815243808, e-mail: brodzkiadam@op.pl

Key Words: Dog, perianal gland tumors, tamoxifen, DNA adduct, genotoxicity. and neoplastic progression are observed in adult and elderly dogs quite frequently, due to side-effects of hormonal stimulatory action. Among all observed neoplastic changes, the most frequent are hepatoid gland adenomas and hepatoid gland adenocarcinomas located in the skin of the perianal region. Contrary to males, perianal glands in females undergo gradual regression with age. The exact etiology of perianal gland tumors incidence has not been explained yet; however, there are relationships between their occurrence, sex and hormonal status $(2,3)$. According to epidemiological data, the perianal gland tumors contribute to $9-18 \%$ of the total neoplastic changes of the skin in dogs (4-7).

DNA adducts are considered as markers of genetic defects. Exposure of DNA to physical factors such as ultraviolet light and ionizing radiation, as well as endogenous and exogenous chemical substances, including hormones, may lead to DNA adduct formation (8). Sexual hormones may stimulate neoplastic development due to stimulation of cellular proliferation processes. Estrogens may lead to DNA damage and DNA adduct formation, leading to carcinogenesis $(9,10)$. Contrary to these findings, other studies have shown that estrogens have a protective role against carcinogenesis. It has been shown that treatment with estrogen or castration of dogs suffering from adenoma lead to partial or total neoplastic remission (11). The protective role of estrogens may be postulated based on data showing perianal gland tumor occurrence mainly in bitches previously subjected to ovariohysterectomy, in which low estrogen levels were not effective in inhibiting neoplastic proliferation (12). In addition, lower occurrence of perianal gland tumors has been observed in dogs suffering from testicular sertolioma showing estrogenic activity (3). The protective role of estrogens against neoplastic proliferation might be also postulated the increased incidence of perianal gland tumors in spayed bitches (12). Lower estrogen release due to ovariohysterectomy, decreases blood estrogen levels and 
increases female susceptibility to perianal gland tumors. Sexual dimorphism of perianal gland cells may be postulated, since atrophy of these cells to single islets has been observed in adult and elder females, while in adult males cellular masses are diagnosed $(13,14)$.

Chemical substances present in pollutants of the natural environment such as polycyclic aromatic hydrocarbons, mycotoxins, aromatic and heterocyclic amines, as well as substances contained in tobacco amines are considered key players in the etiopathogenesis of perianal gland tumors. Most of these substances may form covalent bonds with nucleophilic parts of the DNA chain and induce DNA adduct formation $(15,16)$. Thus, studies on covalent DNA adduct formation as the result of the exposure to endogenous and exogenous toxic factors are becoming increasingly important in the assessment of cumulative environmental hazards affecting the body. Previous studies have highlighted the usefulness of quantifying DNA adduct as a diagnostic marker of the genotoxic effects of various factors $(16,17)$. Diagnostic monitoring of DNA adduct formation may have additional diagnostic value than purely evaluating hazard environmental factors (17). The association between DNA adduct formation and neoplastic tumor development has been underlined in many previous studies $(8,18)$. However, occurrence of DNA adduct does not necessarily indicate neoplastic process development although neoplastic tissues are characterized by increased DNA adduct formation (19).

The aim of the current study was to determine DNA adduct count in peripheral blood mononuclear cells during antihormonal treatment of benign and malignant perianal gland tumors using tamoxifen. Possible diagnostic monitoring of the effectiveness of the applied antihormonal treatment on the course neoplastic disease in dogs using DNA adduct count determination in peripheral blood mononuclear cells was evaluated.

\section{Materials and Methods}

Experimental design and sampling procedure. The experimental procedures used throughout this study were approved by The II Local Ethics Committee on Animal Experimentation of the University of Life Sciences in Lublin, Poland - reference number 49/2006. The study was performed on male dogs between 8 and 15 years of age which suffered from perianal gland tumors. Five dogs suffered from malignant neoplastic changes such as carcinoma (CAR Group; N=5), 16 dogs experienced epithelioma (EPI Group; $\mathrm{N}=16$ ), and 24 dogs suffered from benign neoplastic changes such as adenoma (ADE Group; $\mathrm{N}=24$ ). The control group of dogs consisted of animals subjected to surgery due to perineal hernia or rectal diverticulum (CTR Group; $\mathrm{N}=25$ ). The experimental and control dogs had not been castrated. All dogs were fed well balanced commercially available diet ad libitum. Full blood samples for DNA adduct assessment were collected at the baseline (before antihormonal treatment) from all dogs, as well as one and six months since the beginning of the antihormonal treatment (during the therapy course in the experimental groups only). The antihormonal (antiestrogenic) treatment was performed with tamoxifeni citras (Tamoxifen, Polfa Kutno, S.A., Poland) using the oral daily dosage of $1 \mathrm{mg} / \mathrm{kg}$ of body weight. The dogs showing serum concentration of $17-\beta$-estradiol equal or higher than $7 \mathrm{ng} / \mathrm{ml}$ were qualified to antiestrogenic treatment. Mononuclear cell isolation from peripheral blood samples was performed using commercial Lymphoprep density medium gradient (STEMCELL Technologies, Köln, Germany). DNA isolation from mononuclear cells was performed using Genomic Prep Plus (A\&A Biotechnology, Gdynia, Poland). The isolated DNA samples were kept in a water solution at $-20^{\circ} \mathrm{C}$ until further analysis. Quantitative determination of DNA adduct count was performed using autoradiography. The autoradiography procedure was performed at $-80^{\circ} \mathrm{C}$ using intensifying screen. Quantitative assessment of the radioactivity of the cleavage pattern containing DNA adduct was performed twice in three-day interval using BAS 2000 Bio-Imaging Analyzer (Fuji Film, Tokyo, Japan). The obtained results were expressed as the DNA adduct count per $10^{9}$ nucleotides.

Statistical analysis. All data are presented as means \pm SEM. Statistical analysis was performed using Statistica software and oneway analysis of variance (ANOVA). Statistically significant differences between the control and experimental groups at the baseline were compared by post hoc Tukey's test for non-equal variables. Statistically significant differences between the baseline values and treatment intervals were compared by Tukey's test for equal variables. For all comparisons $p$-value $<0.05$ was considered as statistically significant.

\section{Results}

The results of DNA adduct evaluation in the control and experimental groups of dogs are shown in the Table I. At the baseline, DNA adduct count reached the highest value in the CTR group than in the ADE and CAR groups, while the DNA adduct count was the lowest in the EPI Group. Statistically significant differences of the DNA adduct count were found between all the evaluated groups at the baseline $(p<0.05)$. DNA adduct count decreased significantly by $46.8 \%$ in the ADE Group after one-month of the antiestrogenic treatment, when compared to the baseline value $(p<0.05)$. DNA adduct count increased significantly in the EPI and CAR groups after one-month anti-hormonal treatment by $21.1 \%$ and $16.7 \%$ compared to the baseline values $(p<0.05)$. DNA adduct count in the ADE group increased significantly after six-month anti-hormonal treatment by $78.7 \%$ and $182.9 \%$ compared to the baseline values and those determined five months earlier $(p<0.05)$. DNA adduct count in the EPI group after the six-month antihormonal treatment increased significantly by $221.5 \%$ and $165.5 \%$ compared to the baseline values and those determined five months earlier $(p<0.05)$. DNA adduct count after the six-month antihormonal therapy in the CAR group increased significantly by $198.3 \%$ and $155.6 \%$ compared to the baseline values and those determined five months earlier $(p<0.05)$. 
Table I. DNA adduct content (DNA adduct count per $10^{9}$ of the nucleotides) in the control dogs and those suffering from adenoma, epithelioma and carcinoma.

\begin{tabular}{|c|c|c|c|}
\hline Group & Baseline & 1-month treatment & 6 -month treatment \\
\hline Control $(\mathrm{N}=25)$ & $35,498^{a} \pm 130$ & - & - \\
\hline Adenoma $(\mathrm{N}=24)$ & $31,591^{\mathrm{bA}_{ \pm 178}}$ & $19,949^{\mathrm{B}} \pm 119$ & $56,441 \mathrm{C}_{ \pm}+96$ \\
\hline Epithelioma $(\mathrm{N}=16)$ & $15,536^{\mathrm{cA}} \pm 324$ & $18,814^{\mathrm{B}} \pm 217$ & $49,951^{C_{ \pm}}+230$ \\
\hline Carcinoma $(\mathrm{N}=5)$ & $17,105^{\mathrm{dA}_{ \pm} 94}$ & $19,963^{B_{ \pm}}+380$ & $51,023 \mathrm{C}_{ \pm} 282$ \\
\hline
\end{tabular}

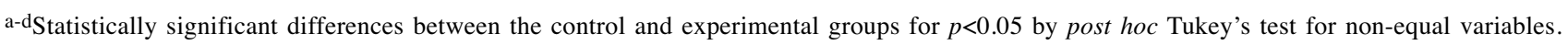
A-CStatistically significant differences between the baseline and treatment intervals for $p<0.05$ by Tukey's test for equal variables.

\section{Discussion}

In his study, the evaluation of DNA adduct count at the baseline has shown the highest value in the control group than in the group of dogs suffering from relatively benign type of tumor - adenoma. In dogs suffering from epithelioma and carcinoma, which are considered as malignant types of tumors, especially carcinoma, the DNA adduct count was relatively low, reaching approximately half of the values determined in the control and ADE groups. The observed huge DNA adduct count in the control and ADE groups seems to be surprising since associations between high rate of DNA adduct formation and neoplastic malignancy and intensive neoplastic disease course (cellular proliferation) has been observed in previous studies (20-23). As reported previously, DNA adduct formation is considered as the earliest genomic change induced by chemical carcinogenesis (24-26). However, there is experimental evidence that DNA adduct formation may constitute an event that is necessary but not sufficient for tumorigenesis. Chronic carcinogen dosing over a range of carcinogen concentrations leads to an early increase in DNA adduct accumulation with time of exposure followed by a plateau observed when the rate of adduct formation is offset by the rate of adduct removal (2629). Based on the results obtained in the current study, it may be postulated that more effective monitoring of tumor development or its antihormonal treatment efficiency would be obtained by analyzing DNA adduct count in tumor target tissues than in peripheral blood mononuclear cells of dogs. This hypothesis is confirmed by previous studies by Boucheron et al. 1987 (28) on rats chronically exposed to diethylnitrosamine in which DNA adduct $\left(O^{4}\right.$ ethyldeoxythymidine) count was evaluated in hepatocyte DNA. $O^{4}$-ethyldeoxythymidine is considered as the most promutagenic adduct associated with the induction of hepatocellular carcinomas (28). Similar findings have been obtained in rats chronically exposed to aflatoxin B1 (30).

Considering DNA adduct count as a marker of antihormonal treatment effectiveness in dogs suffering from benign neoplastic process (adenoma), the initial effects after the first month of therapy with tamoxifen were really impressive since DNA adduct count in mononuclear cells was reduced by nearly $47 \%$. This effect was associated with significant regression of disease symptoms and reduction of perianal tumor volume. Clinical observations confirmed the reduction of the tumor volume approximately by $70-90 \%$ in all dogs from this group. However, in the groups of dogs suffering from malignant tumors (epithelioma and carcinoma), DNA adduct count was not reduced. Moreover, DNA adduct count after one-month antihormonal therapy in these groups increased significantly by $21.1 \%$ and $16.7 \%$, respectively. These observations were surprising because the clinical effects of the antihormonal treatment with tamoxifen in EPI and CAR groups were very promising and clear reduction of the initial tumor volume was observed; however, tumor regression in these groups occurred at a lesser extent than in the ADE group.

In this study, it was shown that 6-month-long antihormonal treatment of dogs with tamoxifen was associated with huge DNA adduct count in mononuclear cells in all the experimental groups of dogs. The baseline values of DNA adduct count was exceeded approximately 2-3 times, indicating the genotoxic effects of tamoxifen in the organism. A genotoxic effect of tamoxifen has been observed in previous studies by HernandezRamon et al. 2014 (31) on female monkey and woman. The equivalent of the daily human therapeutic dose of tamoxifen was given to monkeys (Erythrocebus patas and Macaca fascicularis) for 3-4 months and resulted in tamoxifen-induced DNA adduct formation. Even though liver, uterus and its endometrium seemed to be main target tissues for the genotoxic effects of tamoxifen, DNA adducts were also found in brain cortex and cerebellum. In contrast to the current study, the previous study on monkeys and woman was not focused on DNA adduct evaluation in peripheral blood mononuclear cells, unfortunately. In woman exposed to tamoxifen therapy, but not the control group, DNA adduct formation in endometrium, myometrium and endometrial carcinoma samples was evident (31). The carcinogenic effects of tamoxifen have also been reported in other studies on humans and animals, although it is recommended for adjuvant therapy of breast cancer (32). Our 
results suggest that DNA adduct assessment in mononuclear cells has limited diagnostic value for disease course and treatment effectiveness monitoring. This statement is supported by the clinical observations during the last control visit of the tamoxifen-treated patients, in which the clinical therapeutic effects in all the experimental groups were positive and sufficient for pet owners. To obtain more conclusive diagnostic and prognostic data, long-term assessment of the relationships between neoplastic disease course and antihormonal treatment effectiveness with DNA adduct count evaluation is recommended. Moreover, the analysis of DNA adduct count in dogs undergoing antihormonal treatment, both in the mononuclear cells from the peripheral blood and in tumor target tissue cells would provide more meaningful data. Considering the results obtained in this study, DNA adduct evaluation in peripheral blood mononuclear cells may be considered rather a diagnostic tool for a genotoxic factor present in the body rather than the reference method for the assessment of the effectiveness of antihormonal treatment of neoplastic disease.

In conclusion, this study showed decreased DNA adduct count in mononuclear cells by nearly $47 \%$ in the dogs suffering from adenoma and subjected to one-month antihormonal treatment with tamoxifen. In the groups of dogs suffering from epithelioma and carcinoma an increase of DNA adduct count by $21.1 \%$ and $16.7 \%$, respectively, was observed. Six-month-long therapy with tamoxifen resulted in significant increase of the DNA adduct count by $78.7 \%, 221.5 \%$ and $198.3 \%$ in the ADE, EPI and CAR groups, respectively. The latter data indicating increased DNA adduct formation after long-term administration with tamoxifen, demonstrate the genotoxic effects of tamoxifen. Based on the results obtained in the current study, it may be postulated that more effective monitoring of neoplastic disease course and its antihormonal treatment efficiency would be obtained by analyzing DNA adduct formation in tumor target tissues than in mononuclear cells from the peripheral blood of dogs. However, DNA adduct evaluation in peripheral blood mononuclear cells may potentially be considered as a diagnostic tool for the presence of a genotoxic factor in the body.

\section{Conflicts of Interest}

The Authors declare that they have no conflicts of interest regarding this study.

\section{Authors' Contributions}

Adam Brodzki: designed the study, obtained founding for the study, performed clinical evaluation of patients and collected samples, collected and analyzed data and drafted the manuscript; Marcin Tatara: analyzed data and drafted the manuscript; Piotr Brodzki; collected and analyzed data, critically revised the manuscript; Ireneusz Balicki: analyzed data and critically revised the manuscript.

\section{Acknowledgements}

This study was supported by Grant No NN308295937 from The Polish Ministry of Education and Science.

\section{References}

1 Maita K and Ishida K: Structure and development of the perianal gland of the dog. Jap J Vet Sci 37(4): 349-356, 1975. PMID: 1238859.

2 Goldschmidt MH and Shofer FS: Skin tumors of the dog and cat. Pergamon Press, Oxford, 1992.

3 Brodzki A, Sobczyńska-Rak A, Brodzki P, Tatara MR and Silmanowicz P: Occurrence, etiology and antihormonal treatment of perianal gland tumors in male dogs. Med Weter 70(10): 638-643, 2014.

4 Goldschmidt $\mathrm{M}$ and Hendricks M: Tumours of the skin and soft tissue. In: Tumors in Domestic Animals, 4th edition. Meuten D. (ed.). Ames, Iowa, USA, Iowa State Press, pp. 45-118, 2000. DOI: $10.1002 / 9780470376928 . c h 2$

5 Jakab C, Rusvai M, Glif P and Kulka J: Expression of claudin7 molecule in canine perianal gland tumours. Acta Vet Brno 79(1): 127-133, 2010. DOI: 10.2754/avb201079010127

6 Bray J: Tumours of the perianal region. In: BSAVA Manual of Canine and Feline Oncology, 3rd edition. Dobson JM and Lascelles BDX (eds.). Birmingham, UK, British Small Animal Veterinary Association, pp. 223-228, 2011.

7 Tunc AS, Alcigir ME and Atalay-Vural S: Concurrent metastatic hepatoid gland carcinoma and oesophagogastric leiomyosarcoma in a dog. Ankara Üniv Vet Fak Derg 61(1): 29-34, 2014.

8 Liehr JG, Avitts TA, Randerath E and Randerath K: Estrogeninduced endogenous DNA adduction: possible mechanism of hormonal cancer. Proc Natl Acad Sci USA 83(14): 5301-5305, 1986. PMID: 3460092. DOI:10.1073/pnas.83.14.5301

9 Brodzki A, Łopuszyński W, Brodzki P and Tatara MR: Diagnostic and prognostic value of cellular proliferation assessment with $\mathrm{Ki}-67$ protein in dogs suffering from benign and malignant perianal tumors. Folia Biol (Krakow) 62(3): 235-241, 2014. PMID: 25412511. DOI: 10.3409/fb62_3.235

10 Belous AR, Hachey DL, Dawling S, Roodi N and Parl FF: Cytochrome P450 1B1- mediated estrogen metabolism results in estrogen-deoxyribonucleoside adduct formation. Cancer Res 67(2): 812-817, 2007. PMID: 17234793. DOI: 10.1158/00085472.CAN-06-2133

11 Wilson GP and Hayes HM: Castration for treatment of perianal gland neoplasms in the dog. J Am Vet Med Assoc 174(12): 1301-1303, 1979. PMID: 511727.

12 Millán Y, Guil-Luna S, Reymundo C, Sánchez-Céspedes R and de las Mulas JM: Sex steroid hormones and tumors in domestic animals. In: Insights from Veterinary Medicine. Payan Carreira R (ed.). London, UK, IntechOpen, pp. 191-214, 2013. DOI: $10.5772 / 54324$

13 Shabadash SA and Zelikina TI: The sex dimorphism of the hepatoid circumanal glands in the dog and the dynamics of its development. Izv Akad Nauk Ser Biol 5: 590-605, 1995. PMID: 8520438 .

14 Tozon N, Kodre V, Sersa G and Cemazar M: Effective treatment of perianal tumors in dogs with electrochemotherapy. Anticancer Res 25(2A): 839-846, 2005. PMID: 15868917. 
15 Miller EC and Miller JA: Mechanisms of chemical carcinogenesis. Cancer 47(5 Suppl): 1055-1064, 1981. PMID: 7016297.

16 Beach AC and Gupta RC: Human biomonitoring and the 32Ppostlabeling assay. Carcinogenesis 13(7): 1053-1074, 1992. PMID: 1638670.

17 Uziel M, Munro NB, Katz DS, Vo-Dinh T, Zeighami EA, Waters MD and Griffith JD: DNA adduct formation by 12 chemicals with populations potentially suitable for molecular epidemiological studies. Mutat Res 277(1): 35-90, 1992. PMID: 1376441.

18 Liehr JG: Role of DNA adducts in hormonal carcinogensis. Regul Toxicol Pharmacol 32(3): 276-282, 2000. PMID: 11162721. DOI: $10.1006 /$ rtph.2000.1432

19 Verna L, Whysner J and Williams GM: 2-acetyloaminofluorene mechanistic data and risk assessment: DNA reactivity, enhanced cell proliferation and tumor initiation. Pharmacol Ther 71(1-2): 83105, 1996. PMID: 8910950. DOI:10.1016/0163-7258(96)00063-0

20 Belinsky SA, Walker VE, Maronpot RR, Swenberg JA and Anderson MW: Molecular dosimetry of DNA adduct formation and cell toxicity in rat nasal mucosa following exposure to the tobacco specific nitrosamine 4-( $N$-methyl- $N$-nitrosamino)-1-(3pyridyl)-1-butanone and their relationship to induction of neoplasia. Cancer Res 47(22): 6058-6065, 1987. PMID: 3664508.

21 Deal FH, Richardson FC and Swenberg JA: Dose response of hepatocyte replication in rats following continuous exposure to diethylnitrosamine. Cancer Res 49: 6985-6988, 1989. PMID: 2582440.

22 Belinsky SA, Foley JF, White CM, Anderson MW and Maronpot RR: Dose-response relationship between $O^{6}$-methylguanine formation in Clara cells and induction of pulmonary neoplasia in the rat by 4-(methylnitrosamino)-1-(3-pyridyl)-1-butanone. Cancer Res 50(12): 3772-3780, 1990. PMID: 2340522.

23 Poirier MC and Beland FA: DNA adduct measurements and tumor incidence during chronic carcinogen exposure in rodents. Environ Health Perspect 10: 161-165, 1994. PMID: 7889840. DOI:10.1289/ehp.94102s6161

24 Miller JA: Carcinogenesis by chemicals: an overview - G. H. A. Clowes memorial lecture. Cancer Res 30(3): 559-576, 1970. PMID: 4915745.
25 Miller EC: Some current perspectives on chemical carcinogenesis in humans and experimental animals: Presidential Address. Cancer Res 38(6): 1479-1496, 1978. PMID: 348302.

26 Poirier MC, Hunt JM, True BA, Laishes BA, Young JF and Beland FA: DNA adduct formation, removal and persistence in rat liver during one month of feeding 2-acetylaminofluorene. Carcinogenesis 5(12): 1591-1596, 1984. PMID: 6499111.

27 Belinsky SA, White CM, Boucheron JA, Richardson FC, Swenberg JA and Anderson M: Accumulation and persistence of DNA adduct in respiratory tissue of rats following multiple administrations of the tobacco specific carcinogen 4-(N-methyl$\mathrm{N}$ nitrosamino)-1-(3-pyridyl)-1-butanone. Cancer Res 46(3): 1280-1284, 1986. PMID: 3943097.

28 Boucheron JA, Richardson FC, Morgan PH and Swenberg JA: Molecular dosimetry of O4-ethyldeoxythymidine in rats continuously exposed to diethylnitrosamine. Cancer Res 47(6): 1577-1581, 1987. PMID: 3815358.

29 Yuspa SH and Poirier MC: Chemical carcinogenesis: from animal models to molecular models in one decade. Adv Cancer Res 50: 25-70, 1988. PMID: 3287845. DOI: 10.1016/S0065230X(08)60434-0

30 Wild CP, Garner RC, Montesano R and Tursi F: Aflatoxin B1 binding to plasma albumin and liver DNA upon chronic administration to rats. Carcinogenesis 7(6): 853-858, 1986. PMID: 3085966.

31 Hernandez-Ramon EE, Sandoval NA, John K, Cline JM, Wood CE, Woodward RA and Poirier MC: Tamoxifen-DNA adduct formation in monkey and human reproductive organs. Carcinogenesis 35(5): 1172-1176, 2014. PMID: 24501327. DOI:10.1093/carcin/bgu029

32 Phillips DH: Understanding the genotoxicity of tamoxifen? Carcinogenesis 22(6): 839-849, 2001. PMID: 11375888.

Received March 8, 2019

Revised April 4, 2019

Accepted April 8, 2019 